\title{
Motion prediction in MRI-guided radiotherapy based on interleaved orthogonal cine-MRI
}

\author{
M Seregni ${ }^{1}$, C Paganelli ${ }^{1}$, D Lee ${ }^{2}$, P B Greer ${ }^{3,4}$, G Baroni $^{1,5}$, P J Keall ${ }^{2}$ \\ and M Riboldi ${ }^{1,5}$ \\ ${ }^{1}$ Dipartimento di Elettronica, Informazione e Bioingegneria, Politecnico di Milano, Milano, Italy \\ 2 Radiation Physics Laboratory, Sydney Medical School, University of Sydney, Sydney, Australia \\ ${ }^{3}$ School of Mathematical and Physical Sciences, The University of Newcastle, Newcastle, NSW, \\ Australia \\ ${ }^{4}$ Department of Radiation Oncology, Calvary Mater Newcastle, Newcastle, NSW, Australia \\ 5 Bioengineering Unit, Centro Nazionale di Adroterapia Oncologica, Pavia, Italy
}

\begin{abstract}
In-room cine-MRI guidance can provide non-invasive target localization during radiotherapy treatment. However, in order to cope with finite imaging frequency and system latencies between target localization and dose delivery, tumour motion prediction is required. This work proposes a framework for motion prediction dedicated to cineMRI guidance, aiming at quantifying the geometric uncertainties introduced by this process for both tumour tracking and beam gating. The tumour position, identified through scale invariant features detected in cine-MRI slices, is estimated at highfrequency $(25 \mathrm{~Hz})$ using three independent predictors, one for each anatomical coordinate. Linear extrapolation, auto-regressive and support vector machine algorithms are compared against systems that use no prediction or surrogate-based motion estimation. Geometric uncertainties are reported as a function of image acquisition period and system latency. Average results show that the tracking error RMS can be decreased down to a $[0.2 ; 1.2] \mathrm{mm}$ range, for acquisition periods between 250 and $750 \mathrm{~ms}$ and system latencies between 50 and $300 \mathrm{~ms}$. Except for the linear extrapolator, tracking and gating prediction errors were, on average, lower than those measured for surrogate-based motion estimation. This finding suggests that cine-MRI guidance, combined with appropriate prediction algorithms, could relevantly decrease geometric uncertainties in motion compensated treatments.
\end{abstract}

Keywords: MRI guidance, tumour motion prediction, tumour tracking, beam gating

This is the Accepted Manuscript version of an article accepted for publication in Physics in Medicine and Biology, 2016, 61:2, 872-887. IOP Publishing Ltd is not responsible for any errors or omissions in this version of the manuscript or any version derived from it. The Version of Record is available online at: http://dx.doi.org/10.1088\% 2F0031-9155\%2F61\%2F2\%2F872 


\section{Introduction}

Over the last few years, there has been growing interest in the use of Magnetic Resonance Imaging (MRI) in image guided radiotherapy (Hugo et al 2012, Liney et al 2014). The excel-lent soft-tissue contrast and the absence of ionizing radiation suggest the use of MRI as an attractive technology for target localization. Moreover, its fast (cine) sequences allow studying respiratory organ motion (Biederer et al 2010, Lagendijk et al 2014a) but, due to the trade-off between spatial and temporal resolution (Plathow et al 2009), the real-time acquisition of $3 \mathrm{D}$ target volumes is still a challenge. In previous studies, organ motion was quantified by using cine-MRI sequences for the acquisition of parallel or orthogonal slices (Koch et al 2004, Liu et al 2004, Plathow et al 2004, Dowling et al 2014, Paganelli et al 2015a). Specifically, Koch et al (2004) acquired fast MR images with an acquisition time of $450 \mathrm{~ms}$, whereas Plathow et al (2004) reported cine-MR imaging of lung cancer patients at $\sim 3$ frames per second (fps) (i.e. $300 \mathrm{~ms}$ per image). A higher frame rate can be achieved through the use of accelerating factors, as proposed by Plathow et al (2005) and Sawant et al (2014), reaching up to $6 \mathrm{fps}$ (i.e. $\approx 150 \mathrm{~ms}$ per image). By using such imaging modali-ties, interleaved orthogonal (sagittal and coronal) slices can be acquired at frequencies that are still adequate to monitor the respiratory motion, thus allowing the reconstruction of the 3D tumour position over time (Bjerre et al 2013, Tryggestad et al 2013, Brix et al 2014). Furthermore, the better contrast in MR images with respect to X-ray projections could also avoid the implantation of fiducial markers, and lead to a marker-less identification of internal structures. Common localization approaches rely on template matching of a region of interest (Bjerre et al 2013, Brix et al 2014), whereas other solutions proposed the tracking of robust image features, as reported by Paganelli et al (2015a). These advantages of MRI motivated several recent technological developments towards the integration of MRI with radiation therapy treatment units, raising the prospect of fully MRI-guided treatments (Fallone 2014, Kupelian and Sonke 2014, Keall et al 2014a, Lagendijk et al 2014b, Mutic et al 2014). A key potential improvement in MRI-guidance is the ability to provide time-resolved infor-mation for in-line motion compensation protocols. Currently, the first implementation of an MRI-guided system (Mutic et al 2014) delivers radiotherapy using a gated approach for lung cancer patients, i.e. irradiating in a specific breathing phase, ensuring that the intended dose of radiation is delivered to the tumour and that normal tissues and other critical structures are spared. However, the existing results on $\mathrm{x}$ ray-guided radiotherapy report that enhanced accuracy and tissue sparing can be achieved by adjusting the beam position to follow the tumour motion in real-time (i.e. tumour tracking) (Keall et al 2001, Kilby et al 2010, Verellen et al 2010, Keall et al 2014).

Preliminary works on tumour tracking in MRI studied the use of navigator signals at diaphragm (upper liver dome scout) as surrogates to track tumour motion. These signals provide high temporal resolution $(15 \mathrm{~ms})$ on a $1 \mathrm{D}$ scale and have been evaluated first for gated delivery (Crijns et al 2011) then for real-time tracking (Crijns et al 2012). However, tracking 3D tumour motion and deformations can be difficult with this approach due to the 1D signal extracted from clinically available navigators. These limitations can be overcome through the interleaved acquisition of orthogonal slices intersecting on the target, which can provide 
3D target tracking during irradiation (Bjerre et al 2013, Tryggestad et al 2013, Brix et al 2014). However, similarly to $\mathrm{x}$-ray imaging (Sharp et al 2004), real-time motion compensation with cine-MRI requires time for the execution of the specific pulse sequences, the post processing of raw data, and the reaction time of dose delivery hardware such as the multi leaf collimator. These processes require an amount of time that can lead to localization errors if not properly compensated. In addition, image acquisition periods, even if as short as $150 \mathrm{~ms}$, do not provide true real-time information on the tumour position. As a consequence, motion prediction methods can be expected to play a relevant role in cine-MRI guided treatments. Different prediction strategies were proposed in the literature, mainly focusing on x-ray guided radiotherapy. Part of these studies applied prediction to signals relative to patient body surface motion (external surrogates), which are then used to drive an internal-external correlation model estimating the actual tumour position (Murphy et al 2006, Ernst et al 2009, Ruan 2010). Conversely, other contributions focused directly on the internal tumour motion, detected by means of fluoroscopy, and simulated different combinations of acquisition periods and latencies (Sharp et al 2004, Krauss et al 2011). In addition, all commercial devices implementing surrogate-based motion tracking, such as CyberKnife Synchrony ${ }^{\circledR}$ RTS (Accuray Inc., Sunnyvale, CA) (Kilby et al 2010) and VERO ${ }^{\mathrm{TM}}$ System (Brainlab AG, Feldkirchen, Germany) (Depuydt et al 2013), rely on motion prediction to compensate for latencies (115 ms for the Synchrony ${ }^{\circledR}$ RTS and $50 \mathrm{~ms}$ for the VERO ${ }^{\text {TM }}$ System). However, the direct translation of prediction methods developed for $\mathrm{x}$-Ray image guidance to MRI guided treatments can be difficult due to the different imaging modalities (i.e. interleaved orthogonal slices instead of stereo x-Ray images) as well as to longer acquisition periods (e.g. $150 \mathrm{~ms}$ instead of $30 \mathrm{~ms} x$-Ray fluoroscopy (Shirato et al 2000)) and overall system latencies (up to $300 \mathrm{~ms}$, Mutic et al 2014).

Therefore, the aim of this work is to investigate tumour motion prediction approaches dedicated to MRI-guided treatments based on the acquisition of interleaved orthogonal slices. For this purpose, we relied on cine-MR image sequences acquired with a $3 \mathrm{~T}$ clinical scanner. First, a general workflow, specific for this imaging modality, is proposed. Within this framework, different algorithms are tested and their geometric accuracy, expressed as the difference between predicted and real tumour position, is measured as a function of image acquisition period and system latency. Prediction errors are then compared against those measured when no prediction is applied and against the errors measured by simulating surrogate-based motion tracking instead of cine-MRI guidance. The expected outcome is a quantification of the geometric uncertainties introduced by tumour motion prediction in MRI guided treatments, both for real-time tumour tracking and beam gating.

\section{Materials and methods}

\subsection{Dataset}

Six lung cancer patients underwent MR imaging with a 3.0 T Skyra scanner (Siemens) at the Calvary Mater Hospital, Newcastle. The cine-MRI sequence (balanced steady-state free precession, TrueFISP) included the interleaved acquisition of 512 coronal and 512 sagittal slices, with an acquisition period of $250 \mathrm{~ms}$ per slice, i.e. two coronal and two sagittal slices per second. Therefore, 1024 frames were acquired in a time period of about $256 \mathrm{~s}$. The main MR parameters are reported hereafter:

- repetition time/echo time: $3.03 \mathrm{~ms} / 1.32 \mathrm{~ms}$.

- flip angle: $45^{\circ}$. 
- bandwidth: $1500 \mathrm{~Hz}$ per pixel.

- scan matrix: $256 \times 256$ pixels with $1.48 \times 1.48 \mathrm{~mm}$ spacing.

- slice thickness: $5 \mathrm{~mm}$.

- k-space percentage sampling: $65 \%$

- Acceleration factor: 2 (i.e. two channel parallel imaging)

All image datasets were acquired in free breathing, except for patient 4 for whom audio-visual feedback was used and no free breathing acquisition was available. Patients 1 had a second cine-MRI repeated $5 \mathrm{~min}$ after the first one, whereas patient 5 underwent a second scan after $21 \mathrm{~d}$. Consequently, eight acquisitions were considered in this work.

\subsection{Tumour trajectory extraction}

A custom algorithm based on the Scale Invariant Features Transform (SIFT) (Lowe 2004), was used to detect and track the position of multiple features in cine-MR images. The algorithm consists of two main steps. First, a set of landmarks (referred to as 'features') is extracted from a reference image (first sample of the cine-MRI series) and from a target image (each subsequent cine-MRI slice) based on local contrast. Then a matching algorithm is used to define correspondences between these two features sets. Such a matching algorithm is implemented by measuring the Euclidean distance between the so-called features descriptors, which encode the local image information in terms of intensity and gradient magnitude/direction. The feature extraction method was previously described in Paganelli et al (2013) and applied to cineMRI data in Paganelli et al (2015a).

The above described procedure was performed offline, i.e. on the all slices before applying prediction. Coronal slices provided the measurement of features positions in Left-Right (LR) and Superior-Inferior $\left(\mathrm{SI}^{\mathrm{cor}}\right)$ directions with a sampling period of $500 \mathrm{~ms}$, whereas the Anterior-Posterior (AP) motion, combined with an additional measurement of the SI position $\left(\mathrm{SI}^{\mathrm{sag}}\right)$, was derived from sagittal slices at the same frequency. Since the SI tumour position was measured on both slice orientations, its trajectory (obtained by averaging $\mathrm{SI}^{\mathrm{cor}}$ and $\mathrm{SI}^{\mathrm{sag}}$ ) was sampled at twice the frequency with respect to the two other coordinates (i.e. with a sampling period of $250 \mathrm{~ms}$ instead of $500 \mathrm{~ms}$ ).

Only features detected in the tumour area were considered and their trajectories were averaged to define the overall tumour trajectory. In order to quantify the overall tumour position uncertainty observed during the cine-MRI acquisition, the range of motion of each trajectory was measured (table 1), thus considering both the respiratory motion amplitude as well as baseline drifts.

\subsection{External surface motion}

During the cine-MRI acquisition, external thoraco-abdominal motion was measured by means of a Physiological Measurement Unit (PMU, 3T Skyra, Siemens Healthcare Erlangen, Germany) and the Real-Time Position Management ${ }^{\mathrm{TM}}$ (RPM, Varian Medical Systems, Inc., Palo Alto, CA). The PMU signal was not directly used as external surrogate since its amplitude is constantly normalized by the acquisition software, thus not measuring the real surface displacement, as instead provided by the RPM signal. Therefore, since the RPM system did not share a common time reference with the MR scanner, temporal correspondence between its signal and slices acquisition times was derived by applying a phase matching algorithm to maximize the correlation (i.e. minimize the phase shift) between the RPM $(25 \mathrm{~Hz})$ and the PMU $(400 \mathrm{~Hz})$ signals, the latter being fully 
Table 1. Tumour range of motion and mean respiratory period in the considered acquisitions. The number of tumour features detected in each image sequence (coronal and sagittal) is also reported.

\begin{tabular}{llllrl}
\hline & & \multicolumn{3}{l}{ Tumour range of motion $(\mathrm{mm})$} & \\
\cline { 3 - 4 } & \#Features & & & \\
Patient & (COR-SAG) & LR & AP & SI & Pean \\
\hline P01-1 & $2-4$ & 3.0 & 8.1 & 5.2 & 7.8 \\
P01-2 & $6-9$ & 2.3 & 3.6 & 6.4 & 7.3 \\
P02 & $6-3$ & 1.6 & 5.8 & 8.8 & 5.5 \\
P03 & $6-6$ & 2.2 & 3.4 & 3.6 & 4.8 \\
P04 & $7-14$ & 3.2 & 2.0 & 3.5 & 5.1 \\
P05-1 & $3-3$ & 4.5 & 2.7 & 20.5 & 4.1 \\
P05-2 & $7-4$ & 5.9 & 2.2 & 32.3 & 5.7 \\
P06 & $4-9$ & 3.6 & 3.8 & 3.7 & 3.6 \\
\hline
\end{tabular}

synchronized with the scanner. A unique correlation peak was detected in each dataset, with a median Pearson $\mathrm{r}$ coefficient equal to 0.9 (1st and 3rd quartiles $=0.62$ and 0.92 , respectively).

\subsection{Real-time tumour motion prediction workflow}

The proposed prediction method was designed to provide a real-time estimation of the 3D tumour position at an arbitrary high sample rate, regardless of the cine-MRI acquisition time, the interleaved measurements of the tumour position and the possible system latencies. Figure 1 outlines the workflow of the proposed method.

Three independent (i.e. coordinate-specific) predictors were considered as in Kilby et al (2010) and Depuydt et al (2013) (red, cyan and green flowcharts in figure 1, respectively). In this specific application, such approach allowed to cope with the different sampling rates and the interleaved measurement of each motion coordinate. Initial training entailed the optimization of each predictor's parameters, relying on the first $20 \mathrm{~s}$ of the ground truth tumour motion measured from each cine-MRI sequence. After the initial training, real-time operation was simulated as detailed in figure 1 (panels B1 and B2).

Considering a generic motion coordinate $(x)$, linear interpolation was applied between its last known sample $(x(k))$, measured in the $k$-th cine-MRI frame, and the predicted next sample $(\widehat{x}(k+1))$, obtaining an arbitrary high frequency signal $\widehat{x}(t)(25 \mathrm{~Hz}$, consistently with the RPM signal).

When a new image is available $(t=k+1)$ the predictor's parameters are re-optimized after inserting the new sample into the training datasets with a first-in-first-out logic, then $\widehat{x}(k+2)$ is predicted. Finally, the interpolator is updated to provide the real-time signal between $x(k+1)$ and $\widehat{x}(k+2)$.

Such procedure is applied independently for each motion coordinate: prediction and update are triggered in correspondence to each coronal frame for the LR coordinate and to each sagittal frame for the AP coordinate, i.e. at half of the cine-MRI acquisition rate. As the SI coordinated is measured on both coronal and sagittal slices, its predictor and interpolator are updated in correspondence to every image, i.e.at the full acquisition rate.

Six imaging acquisition periods and six system latencies were simulated to test the proposed method in different scenarios. 


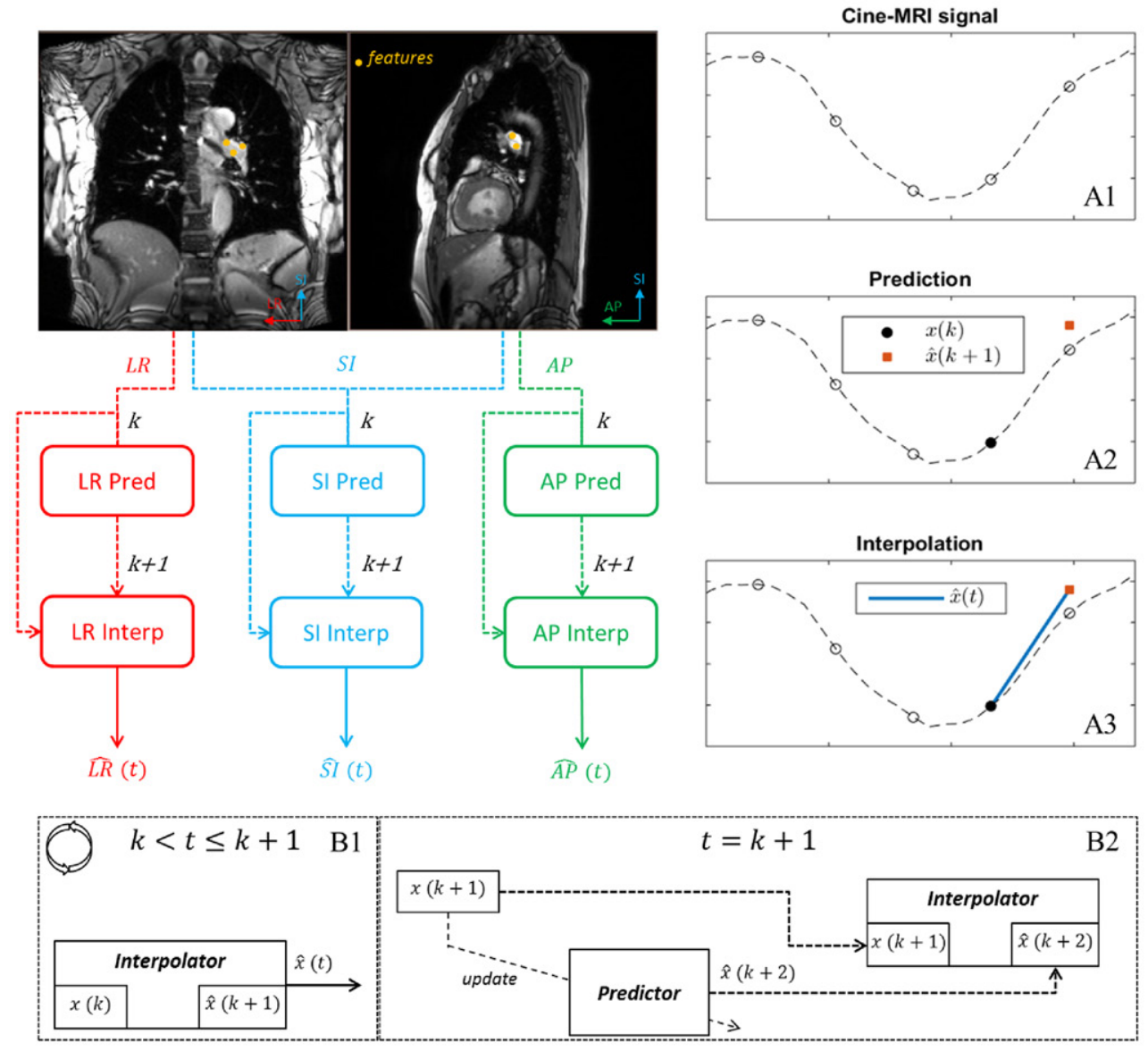

Figure 1. Left panel: workflow of the prediction and interpolation method. Red, cyan and green represent LR, SI and AP motion coordinates, respectively. Dashed lines refer to low frequency signals (i.e. sampled at the cine-MRI acquisition period), whereas solid lines indicate high frequency (i.e. real time) respiratory signals. Panels A1 to A3: exemplification of the cine-MRI signal, prediction of the $k+1$ sample at time $\mathrm{k}$, interpolation between the $k$-th sample and the predicted $\mathrm{k}+1$ sample. Data shown represents a 3s-long respiratory act sampled at $750 \mathrm{~ms}$ period. Panel B1 and B2: realtime operation of the prediction and interpolation method: high frequency interpolation (B1), prediction and update procedures (B2).

- Acquisition periods $(\Delta T)$ from 250 to $750 \mathrm{~ms}$ with $100 \mathrm{~ms}$ interval were simulated by resampling the original motion signals. Higher values of $\Delta T$ were not considered as they would have resulted in inconsistent sampling of the LR and AP coordinates in presence of respiratory acts shorter than $3 \mathrm{~s}$.

- The system latency $(\Delta L)$ was simulated by triggering the update procedure at time $t^{\prime}=t_{k}+\Delta L$, where $t_{k}$ is the acquisition time of a given slice. In this way, as discussed by Sharp et al (2004), the tumour position estimation between $t^{\prime}=t_{k}+\Delta L$ and $t^{\prime \prime}=t_{k}+\Delta T+\Delta L$ was based on the information retrieved from the $k$-th cine-MRI slice. The range of simulated latencies was chosen according to values reported in the literature: from $50 \mathrm{~ms}$ (VERO ${ }^{\mathrm{TM}}$ System (Depuydt et al 2013)) up to $300 \mathrm{~ms}$ (ViewRay System (Mutic et al 2014)) with a $50 \mathrm{~ms}$ step. 


\subsection{Prediction algorithms}

First of all, the scenario where no prediction is applied to the tumour motion trajectories was simulated by imposing $\widehat{x}(k<t \leqslant k+1)=x(k)$. Then, three different prediction models were implemented.

- Linear extrapolation, which assumes that the signal keeps a constant speed during the entire prediction horizon. It requires neither parameter optimization nor structure selection.

- Autoregressive linear prediction (AR): the predicted tumour position is obtained as a linear combination of $h$ previous samples (histories) of the same signal (Sharp et al 2004). Its parameters were optimized using the least square method, whereas the number of histories was automatically selected for each dataset as part of the model initial training by minimizing the Akaike Information Criterion (AIC). The minimum number of histories was set to 2 and the maximum to half of the available training samples.

- Support Vector Machines (SVM) is a class of machine learning methods able to solve linear and non-linear classification and regression problems. In this work they were applied as time-series predictors as proposed by Ernst et al (2009). In order to implement a non-linear predictor, the Gaussian Radial Basis Function kernel was selected. The $\epsilon$ parameter (i.e. fitting error tolerance) was set to 0.1 , corresponding to $10 \%$ of the range of motion of the training data, which were remapped in the $[0,1]$ range. The two other SVM-specific parameters, which are $C$ (penalty factor) and $\gamma\left(=1 / 2 \sigma^{2}, \sigma=\right.$ standard deviation of the Gaussian kernel), were selected during the initial training using a grid search algorithm.

\subsection{Internal/external correlation}

Along with the tumour motion temporal prediction, an internal-external correlation model was implemented relying on the RPM signal as external surrogate. This approach differs from the temporal prediction as the tumour motion is inferred as a function of an external surrogate, instead of relying solely on previous values. We tested this method as it represents the state of the art in tumour motion compensation (Kilby et al 2010, Depuydt et al 2013). Similarly to our previous work (Paganelli et al 2015a), a quadratic state augmented internal-external cor-relation model was considered, as reported in the following equation:

$$
\hat{x}(t)=a s^{2}(t)+b s(t)+c+d \dot{s}^{2}(t)+e \dot{s}(t)
$$

where the target position along a given motion direction $(\widehat{x}(t))$ is estimated as a quadratic function of the position and velocity of the external surrogate $(s(t)$ and $\dot{s}(t)$, respecivelly), i.e. the RPM signal. Its parameters $[a, b, c, d, e]$ were optimized with the least square method on a training dataset collected during the first $20 \mathrm{~s}$ of the available motion traces. As for the predictors, the update procedure was triggered in correspondence to each new available sample.

\subsection{Geometric uncertainties quantification}

Geometric uncertainties introduced by motion prediction and internal-external correlation were evaluated considering two possible treatment scenarios: (i) tumour tracking, where the estimated signal provided continuous real-time beam steering and (ii) beam gating, where the estimation was used to define the trigger signal for beam on (Shirato et al 2000, Wu et al 2008). 
For the tracking scenario, the uncertainty was quantified as the tracking error (TE), i.e. the Euclidean distance between the predicted and the ground truth tumour position, which is directly measured from cine-MR images. For each dataset, the TE distribution was described by its median, inter-quartile range (IQR), 95th percentile and root mean square (RMS) values. The analysis excluded data used for model training $(\approx 20 \mathrm{~s})$ and therefore was limited to the remaining $\approx 240 \mathrm{~s}$.

Considering beam gating, since the cine-MRI allows direct localization of the tumour without relying on surrogates, a position-based gating method was simulated (i.e. the beam is on when the tumour is detected within a given distance from the planned irradiation position). The irradiation position and the beam-on window size were defined for each dataset and the trade-off between the window size and the duty cycle (the ratio between the beam-on time and the overall treatment time) was established analyzing the Probability Distribution Function (PDF) of the ground truth respiratory signal (Heerkens et al 2014). Specifically, the windows size $(w)$ was set to $30 \%$ of the $3 \mathrm{D}$ range of motion of the tumour and the optimal irradiation position $\left(\overline{\boldsymbol{x}}^{\mathrm{opt}}=[\overline{L R}, \overline{A P}, \overline{S I}]\right)$ was selected by maximizing the duty cycle:

$$
\overline{\boldsymbol{x}}^{\mathrm{opt}}=\operatorname{argmax}_{\overline{\boldsymbol{x}}}\left(\frac{\#\{\sqrt{\|\mathrm{X}-\overline{\boldsymbol{x}}\|} \leqslant w\}}{\#\{\boldsymbol{x}\}}\right)
$$

where $\boldsymbol{x}$ is the tumor position, $\overline{\boldsymbol{x}}$ the irradiation position to be optimized and \# \{.\} represents the number of samples.

The percentage difference (relative to the overall treatment time) between the gating trigger signal calculated on the predicted motion $(\widehat{G}(t))$ and that calculated on the ground truth motion $(G(t))$ was defined as Gating Trigger Difference (GTD). Finally, gating geometric uncertainties (Gating Error, $G E$ ) were quantified as the Euclidean distances between the ground truth

tumour position $(\boldsymbol{x})$ and the irradiation position $\left(\overline{\boldsymbol{x}}^{\mathrm{opt}}\right.$ during the beam-on time defined by $\widehat{G}(t)$. As a reference, the nominal $G E$ was measured considering the gating window defined on the ground truth motion $(G(t))$.

\section{Results}

\subsection{Tracking uncertainties}

As an example, figure 2 shows the high frequency tumour position estimation $(25 \mathrm{~Hz})$ obtained in different scenarios (with or without prediction).

The systematic delay, as observed when no prediction is used, was compensated by the AR prediction. Also the internal-external correlation model compensates for delays but correlation errors can be observed mostly around end-inhale and end-exhale peaks.

Figure 3 reports the RMS of the TE as a function of the acquisition period $(\Delta T)$ and latency $(\Delta L)$. Each measurement is averaged over the eight datasets.

The average TE RMS was limited to $2 \mathrm{~mm}$ for all the considered acquisition periods and latencies. Specifically, linear extrapolation resulted in RMS larger than the no prediction scenario for $\Delta T>550 \mathrm{~ms}$. The AR and the SVM achieved better accuracy with lower sensitivity to acquisition period and latency. Correlation errors were generally larger than all prediction errors, except linear extrapolation errors at $\Delta T>550 \mathrm{~ms}$ and $\Delta L>150 \mathrm{~ms}$. Furthermore, correlation errors were not relevantly influenced by $\Delta T$ and $\Delta L$.

Patient-specific results are reported in table 2, where the 'worst' scenario is considered, i.e. considering the largest acquisition period and latency. 

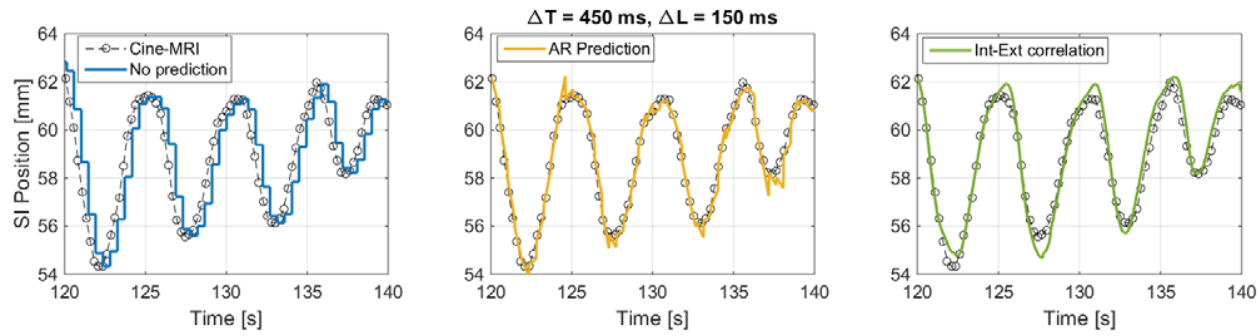

Figure 2. Tumour motion estimation obtained without using prediction (left panel, blue), using AR prediction (center panel, in yellow) and applying the internal-external correlation model (right panel, green). In all panels, the original cine-MRI signal sampled at $250 \mathrm{~ms}$ is reported as dashed black line with circles. Acquisition period and latency are set to $450 \mathrm{~ms}$ and $150 \mathrm{~ms}$ respectively. Data are from patient 2.
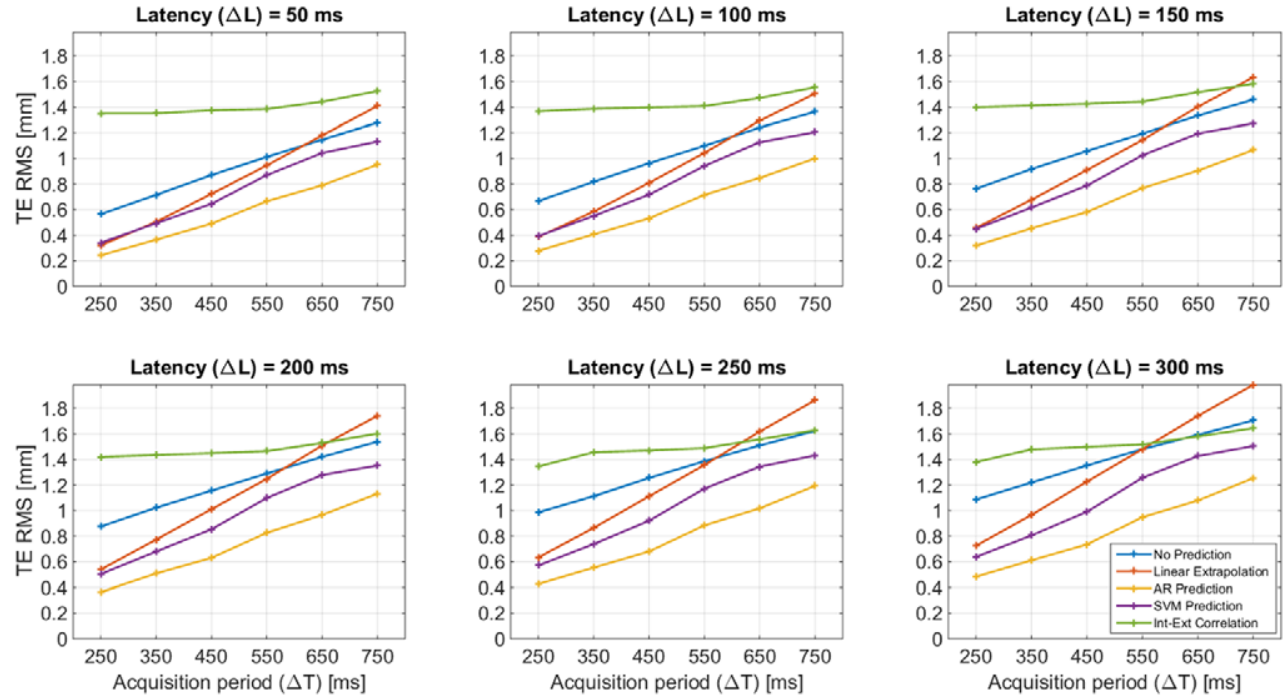

Figure 3. Average TE RMS for each combination of imaging acquisition period and latency.

Table 2. For each available dataset, median, inter-quartile range and 95th percentile of the TE distribution is reported at $\Delta T=750 \mathrm{~ms}$ and $\Delta L=300 \mathrm{~ms}$.

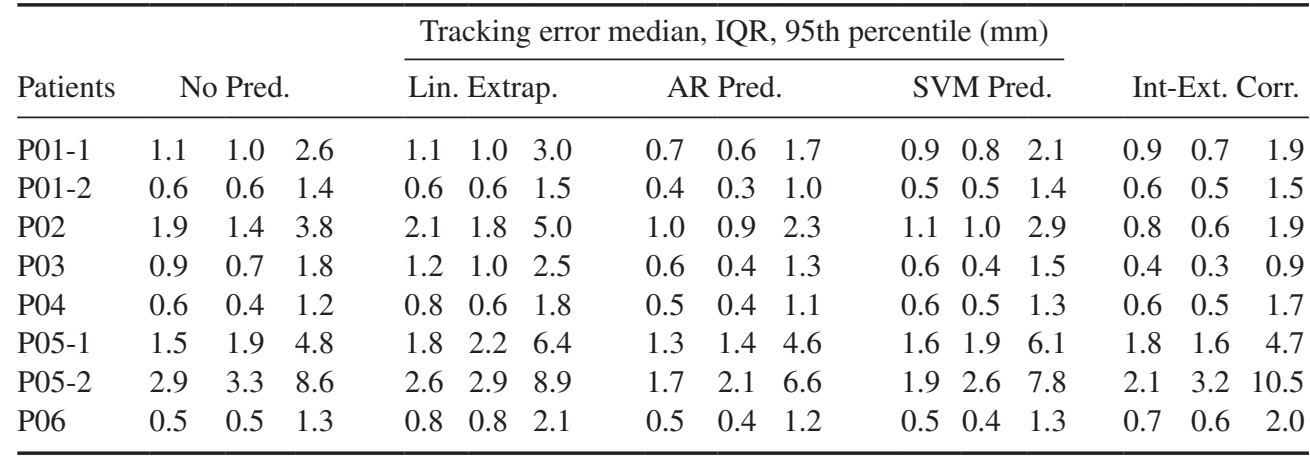



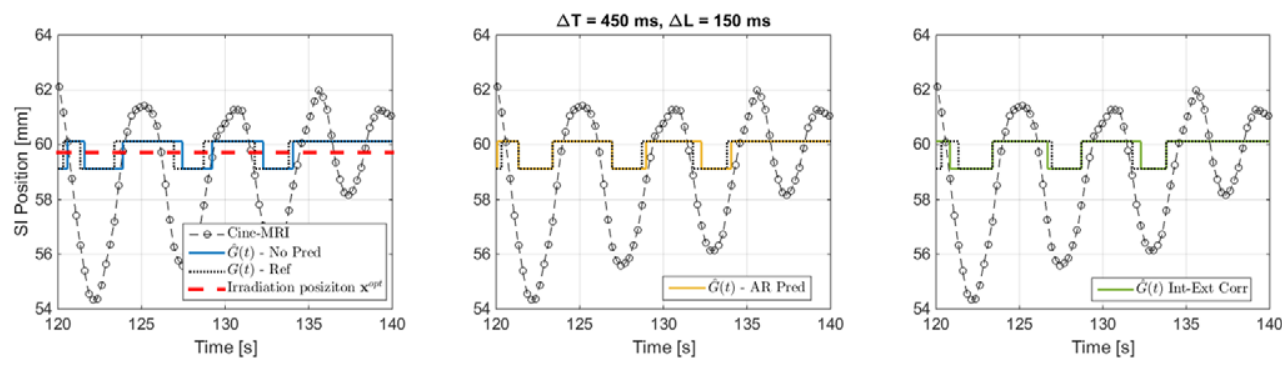

Figure 4. Gating trigger signals calculated for the no prediction scenario (left panel, blue), AR prediction (center, yellow) and internal-external correlation model (right panel, green). The reference trigger signal (i.e. calculated on the ground truth trajectory) is reported as a black dotted line and the red dashed line shows the irradiation position. As in figure 2, data are from patient 2, with $\Delta T=450 \mathrm{~ms}$ and $\Delta L=150 \mathrm{~ms}$.

Tracking errors magnitude varied on a patient by patient basis. Median TE values showed a range of $2.4 \mathrm{~mm}$ for no prediction and $2.0 \mathrm{~mm}$ for linear extrapolation. However, interpatient variability decreased to $1.3 \mathrm{~mm}$ for AR prediction, to $1.4 \mathrm{~mm}$ for SVM prediction and to $1.7 \mathrm{~mm}$ for internal-external correlation. In the 'worst scenario' $(\Delta T=750 \mathrm{~ms}$ and $\Delta L=300 \mathrm{~ms}$ ), the AR predictor achieved the best accuracy in 6 out of 8 datasets, whereas the internal-external correlation showed the lowest median error in patients 2 and 3. Considering the TE RMS as a percentage of each coordinate range of motion (table 1), all predictors achieved the best performance in the SI direction, whose percentage TE RMS was, on average, $6.6 \%$ and $7.0 \%$ lower than those measured on the LR and AP directions, respectively. Significant differences were observed among tracking errors measured with all the methods (Friedman test, $p$-values $<10^{-4}$ ) for $\Delta T=250 \mathrm{~ms}$ and $\Delta L=50 \mathrm{~ms}$. However, in the "worst scenario' non-significant differences (Friedman test, $p$-value $>0.05$ ) were reported between the SVM predictor and the internal-external correlation.

\subsection{Gating uncertainties}

Ground truth $(G(t))$ and predicted $(\widehat{G}(t))$ gating trigger signals are exemplified in figure 4 for no prediction, AR prediction and internal-external correlation.

As expected, the delay observed in figure 2 when no prediction was applied is clearly vis-ible also in the gating trigger signal, which is shifted of about $500 \mathrm{~ms}$ with respect to the reference trigger. Despite local differences between $G(t)$ and $\widehat{G}(t)$, such shift cannot be observed when AR prediction or correlation were applied.

Figure 5 reports the average GTD as a function of image acquisition period and latency. Overall distributions of GE are also reported along with the measured duty cycles.

The Gating Trigger Differences measured using different predictors behaved similarly to the TE reported in figure 3, where the lowest errors are observed for 'complex' predictors (AR and SVM). Considering a latency of $300 \mathrm{~ms}$, the use of the AR predictor allowed reducing the GTD by $9.1 \%$ at $\Delta T=250 \mathrm{~ms}$ and by $5.3 \%$ at $\Delta T=750 \mathrm{~ms}$. Consistently with tracking results, the AR predictor was optimal (i.e. achieved the lowest GTD) in all datasets except for patients 2 and 3, where the internal-external correlation showed the best result (16\% and 11\% with respect to $19 \%$ and $18 \%$ ).

Figure 5(B) reports gating geometric uncertainties by showing the distribution of the Gating Errors for each predictor in the best $(\Delta T=250 \mathrm{~ms}, \Delta L=50 \mathrm{~ms})$ and worst scenarios $(\Delta T$ $=750 \mathrm{~ms}, \Delta L=300 \mathrm{~ms}$ ). All methods (except no prediction) showed median, IQR, and 

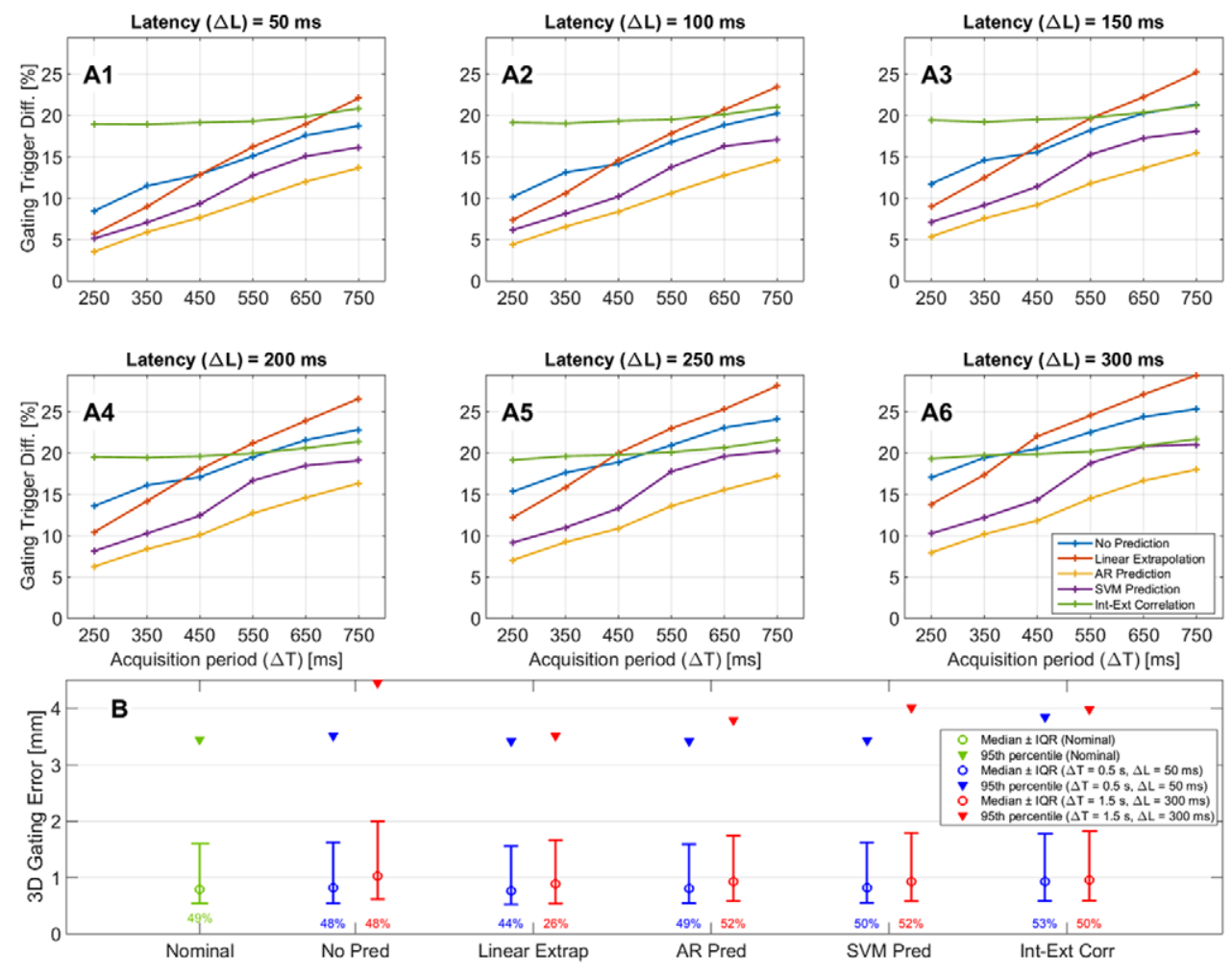

Figure 5. Panel A1 to A6: average GTD for each combination of imaging acquisition period and latency. Panel B: overall distributions of GE. The error bars depict medians and inter-quartile ranges, the triangles represent 95 th percentiles. Data in blue refer to measurements at minimum acquisition period $(250 \mathrm{~ms})$ and latency $(50 \mathrm{~ms})$, whereas data in red refer to maximum period $(750 \mathrm{~ms})$ and latency $(300 \mathrm{~ms})$. Distribution of the nominal gating error, i.e. calculated considering the ground truth trigger signal $(G(t))$, is reported in green. Duty cycles are shown below each error bar.

95th percentile values within $\pm 0.5 \mathrm{~mm}$ with respect to the nominal GE (i.e. measured considering the reference trigger signal). Also their duty cycle differed from the average nominal (49\%) of no more than $4 \%$. A severely decreased duty cycle $(26 \%)$ is measured by applying linear extrapolation at $\Delta T=750 \mathrm{~ms}$ and $\Delta L=300 \mathrm{~ms}$.

\subsection{Computational times}

Computational costs were quantified as the execution time of the prediction and update process (figure 1, panel B2) as well as of the real-time interpolation (figure 1, panel B1). For the internal-external correlation model, as interpolation is not needed, the model update time was considered. All measurements refer to a MATLAB (The MathWorks Inc, Natick, MA) implementation running on a machine equipped with a $3.2 \mathrm{GHz}$ processor and 64GB RAM. The most time consuming process was the update of the AR predictor, which required an average of $105 \mathrm{~ms}$, whereas the SVM predictor and the correlation model computational times were less than $70 \mathrm{~ms}$. Interpolation and linear extrapolation required less than $1 \mathrm{~ms}$. 


\section{Discussion}

The interleaved acquisition of coronal and sagittal cine-MRI slices allows reconstructing the tumour trajectory by measuring its three spatial components. We reported a framework for motion prediction applied to this specific MRI guidance approach.

\subsection{Tumour localization}

Ground truth tumour motion was extracted from the cine-MRI slices using a SIFT-based algorithm (Paganelli et al 2013), which automatically tracked from 6 up to 21 features in the tumour volume (table 1). Feature extraction was performed offline before applying the realtime prediction method. Fast implementations of SIFT-based algorithms that make use of GPU parallel computing are reported to perform feature extraction and matching in less than $40 \mathrm{~ms}$ (Acharya et al 2014, Björkman 2013), therefore the online application of SIFT tumour localization is technically feasible. Nonetheless, the proposed prediction framework can work in combination with any tumour localization approach, such as template matching (Bjerre et al 2013, Brix et al 2014).

In the present study, the image series were acquired with a $3 \mathrm{~T}$ clinical MRI scanner, whereas treatments units currently under development integrate scanner up to $1.5 \mathrm{~T}$. However, the reduced field strength is not expected to hinder feature extraction, as verified in a previous work (Paganelli et al 2015a) where the SIFT-based method was successfully applied on 1.5T cine-MRI series. Furthermore, the relatively lower field strength (e.g. 1.5T rather than 3T) limits magnetic susceptibility artefacts, such as those that may occur at the interface between lung tumours and the surrounding low-density tissues, thus improving features extraction and matching capabilities. Due to the need to optimize the cine-MRI sequences for each specific scanners, also the acquisition period depends on the considered hardware. For this reason, we tested the proposed prediction framework with $\Delta T$ up to $750 \mathrm{~ms}$, thus simulating different scenarios that could be representative of acquisitions performed with different scanners.

By averaging the SI motion coordinates measured in coronal ( $\left.\mathrm{SI}^{\mathrm{cor}}\right)$ and sagittal (SI ${ }^{\mathrm{sag}}$ ) slices, we assumed that, during a cine-MRI guided treatment, the Superior-Inferior motion information can be equivalently derived from both slice orientations. Specifically for the present work, this assumption is supported by the relatively large correlation between $\mathrm{SI}^{\mathrm{cor}}$ and $\mathrm{SI}^{\mathrm{sag}}$ (Pearson $\mathrm{r}$ median $=0.73$, 1st and $3 \mathrm{rd}$ quartiles $=0.50$ and 0.86 , respectively), which proved that the two signals carried essentially the same information. From a general point of view, the features tracked during irradiation should be referred to the isocenter position defined in the treatment plan, therefore obtaining the same information regardless of the slice orientation where they are detected.

\subsection{Tumour motion prediction}

We relied on three independent predictors combined with interpolators, each one generating a high frequency estimation of a single motion coordinate. Since the SI tumour position can be measured on both coronal and sagittal orientations, this approach makes efficient use of the information derived from the interleaved orthogonal cine-MRI: the SI predictor-interpolator is updated at twice the frequency of the two others and operates with half the prediction horizon. Both these factors increase the estimation accuracy on the SI coordinate, which usually showed the largest tumour range of motion (table 1) and therefore caused the most relevant localization uncertainty. 
The prediction algorithms considered in this work represent widely known approaches to tumour motion temporal prediction. Among the large number of prediction algorithms reported in the literature (Sharp et al 2004, Murphy et al 2006, Ernst et al 2009, Ruan 2010, Krauss et al 2011), we tested the linear extrapolation since it is the simplest possible predictor, whereas the AR and the SVM represented more complex linear and nonlinear methods, respectively.

\subsection{Geometric Uncertainties}

The geometric uncertainties of motion prediction and interpolation were quantified by simulating tumour tracking and beam gating. Considering the tracking errors, the performance of the different prediction algorithms can be compared in figure 3 and table 2. On average, the lowest error was achieved by the AR predictor, which generally showed localized inaccuracies $(<1 \mathrm{~mm})$ in correspondence to inhale and exhale peaks, as visible in figure 3 . Such errors were quickly recovered thanks to the adaptive model update method described in figure 1(B2). The relatively worse performance of the SVM predictor suggests that its increased complexity and non-linearity might not be required in this specific application, where the prediction horizon is always within $1.5 \mathrm{~s}$ and the model is constantly updated in correspondence to each cine-MRI slice. A similar behaviour of complex models was also observed in Sharp et al (2004), where the AR predictor outperformed (or was comparable to) more complex predictors such as Artificial Neural Networks and the Kalman filter. As expected, linear extrapolation showed the worse performance.

The sensitivity of each prediction method to the system latency can be observed in figure 3. The no prediction approach was the most sensitive to latency, as its error increased by $0.6 \mathrm{~mm}$ between the minimum $(50 \mathrm{~ms})$ and the maximum $(300 \mathrm{~ms})$ considered latency. The AR pre-dictor was the least affected by this parameter $(0.2 \mathrm{~mm}$ increase $)$. The sensitivity to the image acquisition period $(\Delta T)$ can be also observed in figure 3 , by looking at the positive slope of the RMS curves. The linear extrapolator assumes that the tumour moves with constant velocity, therefore its performances were degraded much faster than other predictors as a function of $\Delta T$, resulting even inferior to the case where no prediction is applied $(\Delta T>550 \mathrm{~ms})$. Since there is a trade-off between temporal and spatial resolution of cine-MRI images (Plathow et al 2009), a low sensitivity to $\Delta T$ allows, in principle, to increase the spatial resolution with-out adding geometric uncertainties due to prediction, thus increasing the overall target locali-zation accuracy. As the native acquisition period was 250 $\mathrm{ms}$ in our dataset, shorter periods were not investigated. However, the introduction of new cine-MRI sequences with advanced parallel imaging and reconstruction strategies could limit the trade-off between temporal and spatial resolution, i.e. by reducing the acquisition period with minimal effects on image qual-ity. For instance, very fast MR sequences (up to $100 \mathrm{~Hz}$ ) were reported for specific applica-tions such as the quantification of very rapid motor function in the oropharyngeal cavity with spatial resolution of $1.5 \mathrm{~mm}$ and field of view of $192 \mathrm{~mm}^{2}$ (Iltis et al 2015). Considering this scenario, results reported in figures 3 and 5 suggest that, for $\Delta T<250 \mathrm{~ms}$, motion prediction would be required to compensate only for system latencies.

With respect to previous works, Sharp et al (2004) predicted the internal tumour displacement detected by means of fluoroscopic imaging. At imaging frequencies within $4 \mathrm{~Hz}$ (i.e. $\Delta T=250 \mathrm{~ms}$ ), the RMS of prediction errors were larger than $1 \mathrm{~mm}$ and $2 \mathrm{~mm}$ at latencies equal to $33 \mathrm{~ms}$ and $200 \mathrm{~ms}$, respectively. Since the errors measured in the no prediction scenario were also larger than those reported in our work, this difference can be partly attributed to the larger breathing amplitudes of the patients but also to the absence of a continuous 
update of the predictors parameters. Even if the results reported by Krauss et al (2011) showed that the adaptive predictor update does not achieve a relevant accuracy improvement (smaller than 5\%), it should be noted that baseline and amplitude drifts were not removed in our work, therefore the adaptive predictor update played a relevant role in preserving the estimation accuracy along the 4 min long cine-MRI scan.

The quadratic internal-external correlation model (Depuydt et al 2013) was implemented to provide an estimation of the accuracy that could be obtained by applying state of the art motion tracking methods instead of the proposed prediction-interpolation based on MRIguidance. In our previous work (Paganelli et al 2015a) this comparison was carried out on bi-dimensional motion traces without considering prediction errors. Results showed that, on average, prediction uncertainties in MRI-guided motion compensation can be expected to be lower than those that can be achieved in surrogate-based motion compensation, even updating the correlation model at periods as short as $250 \mathrm{~ms}$.

The correlation errors measured in this work can be considered comparable to those reported by Mukumoto et al (2014) for the same correlation model (as implemented in the VERO $^{\text {TM }}$ System). However, large errors were observed in patient 5 . They can be attributed to a strongly irregular breathing pattern observed in both its dataset (P05-1 and P05-2) that prevented the accurate fitting of the quadratic internal-external correlation function to the measured data. Since in these datasets the prediction error was relevantly lower than the correlation error, patient 5 may be regarded as an ideal candidate for cine-MRI guidance (Paganelli et al 2015a).

Similarly to tumour tracking, also beam gating will take advantage from non-invasive direct tumour localization achieved by MRI-guidance (Crijns et al 2011, Mutic et al 2014), therefore, geometric uncertainties were quantified also in this scenario.

The Gating Trigger Difference was used to compare the performances of prediction or correlation methods as a function of acquisition period and latency (figure 5(A)), whereas the Gating Error provided a direct quantification of the geometric uncertainties. However, duty cycle should also be considered along with geometric errors (figure 5(B)) to define the overall performance of motion compensation based on beam gating. For instance, the linear extrapolation method, evaluated at $\Delta T=750 \mathrm{~ms}$ and $\Delta L=300 \mathrm{~ms}$ did not show a relevantly increased geometric error with respect to the nominal condition but its duty cycle (i.e. the overall beam on period) was $22.4 \%$ shorter.

Considering the comparative performance of different prediction or correlation methods, the gating errors behaved similarly to tracking errors, thus the same general conclusions can be drawn.

\section{Conclusion}

Motion compensated radiotherapy treatment protocols based on in-room MRI guidance are expected to be clinically implemented in the near future. In this perspective, the main outcome of this work is the development of a tumour motion prediction-interpolation framework specifically designed for MRI guidance. Its most relevant feature is the ability to provide a $3 \mathrm{~d}$ arbitrary high-frequency tumour motion signal from 2D interleaved measurements performed on cine-MR images, while temporal prediction is applied to cope with finite acquisition periods and latencies. In addition, the information on prediction errors as a function of acquisition period and latency allows defining customized treatments safety margins by combining patient-specific variables (e.g. tumour range of motion) with parameters related to the beam delivery hardware and to the in-room image acquisition protocol. 
Considering future developments, the proposed framework can be extended to include the prediction-interpolation of tumour rotations (Paganelli et al 2015b) as well as deformations (Ge et al 2014). Finally, in future scenarios where the acquisition of full 3D volumes will be feasible at adequate temporal and spatial resolution for in-room image guidance, the proposed framework would still be valuable to compensate system latencies, which can be expected to increase due to the time required to acquire and process $3 \mathrm{D}$ instead of $2 \mathrm{D}$ images.

\section{References}

Acharya K A, VenkateshBabu R and Vadhiyar S S 2014 A real-time implementation of SIFT using GPU J. Real-Time Image Process. (doi: 10.1007/s11554-014-0446-6)

Bjerre T, Crijns S, afRosenschöld P M, Aznar M, Specht L, Larsen R and Keall P 2013 3D MRI-linac intra-fraction guidance using multiple orthogonal cine MRI planes Phys. Med. Biol. 58 4943-50

Biederer J, Hintze C, Fabel M and Dinkel J 2010 Magnetic resonance imaging and computed tomography of respiratory mechanics J. Magn. Reson. Imaging 32 1388-97

Björkman M 2013 A CUDA implementation of SIFT, 10 June 2015 (www.csc.kth.se/ celle/)

Brix L, Ringgaard S, Sørensen T S and Poulsen P 2014 3D liver motion tracking using real-time 2D MRI Med. Phys. 41042302

Crijns S P M, Kok J G M, Lagendijk J J W and Raaymakers B W 2011 Towards MRI-guided linear accelerator control: gating on an MRI accelerator Phys. Med. Biol. 56 4815-25

Crijns S P M, Raaymakers B W and Lagendijk J J 2012 Proof of concept of MRI-guided tracked

radiation delivery: tracking 1D motion Phys. Med. Biol. 57 7863-72

Depuydt T et al 2013 Initial assessment of tumour tracking with a gimbaled linac system in clinical circumstances: a patient simulation study Radiother. Oncol. $106236-40$

Dowling J A, Dang K, Chris D F, Chandra S, Gill S, Kron T, Pham D and Foroudi F 2014 Fast cinemagnetic resonance imaging point tracking for prostate cancer radiation therapy planning J. Phys.

Conf. Ser. 489012027

Ernst F and Schweikard A 2009 Forecasting respiratory motion with accurate online support vector regression (SVRpred) Int. J. Comput. Assist. Radiol. Surg. 4 439-47

Fallone B G 2014 The rotating biplanarlinac-magnetic resonance imaging system Semin. Radiat. Oncol. 24 200-2

Ge Y, O'Brien R T, Shieh C C, Booth J T and Keall P J 2014 Toward the development of intrafraction tumor deformation tracking using a dynamic multi-leaf collimator Med. Phys. 41061703

Heerkens H D, VanVulpen M, Van Den Berg C A T, Tijssen R H N, Crijns S P M, Molenaar I

Q, van Santvoort H C, Reerink O and Meijer G J 2014 MRI-based tumour motion characterization and gating schemes for radiation therapy of pancreatic cancer Radiother. Oncol. 111 252-7

Hugo G D and Rosu M 2012 Advances in 4D radiation therapy for managing respiration: part I-4D imaging Med. Phys. 22 258-71

Iltis P W, Frahm J, Voit D, Joseph A A, Schoonderwaldt E and Altenmüller E 2015 High-speed real-

time magnetic resonance imaging of fast tongue movements in elite horn players Quant. Imaging Med. Surg. $5374-81$

Keall P J, Barton M and Crozier S 2014a The Australian magnetic resonance imaging-linac program Semin. Radiat. Oncol. 24 203-6

Keall P J, Colvill E, O'Brien R, Ng J A, Poulsen P, Eade T, Kneebone A and Booth J T 2014b The first clinical implementation of electromagnetic transponder-guided MLC tracking Med. Phys. 41020702

Keall P J, Kini V R, Vedam S S and Mohan R 2001 Motion adaptive x-ray therapy: a feasibility study Phys. Med. Biol. 46 1-10

Kilby W, Dooley J R, Kuduvalli G, Sayeh S and Maurer C R 2010 The cyberknife robotic radiosurgery system in 2010 Technol. Cancer Res. Treat. 9 433-52

Koch N, Liu H H, Starkschall G, Jacobson M, Forster K, Liao Z, Komaki R and Stevens C W 2004 Evaluation of internal lung motion for respiratory-gated radiotherapy using MRI: part I-correlating internal lung motion with skin fiducial motion Int. J. Radiat. Oncol. Biol. Phys. 60 1459-72

Krauss A, Nill S and Oelfke U 2011 The comparative performance of four respiratory motion predictors for real-time tumour tracking Phys. Med. Biol. 56 5303-17 
Kupelian P and Sonke J J 2014 Magnetic resonance-guided adaptive radiotherapy: a solution to the future Semin. Radiat. Oncol. 24 227-32

Lagendijk J J W, Raaymakers B W, Van den Berg C A T, Moerland M A, Philippens M E and van Vulpen M 2014a MR guidance in radiotherapy Phys. Med. Biol. 59 R349-69

Lagendijk J J W, Raaymakers B W and van Vulpen M 2014b The magnetic resonance imaginglinac system Semin. Radiat. Oncol. 24 207-9

Liney G P and Moerland M A 2014 Magnetic resonance imaging acquisition techniques for radiotherapy planning Semin. Radiat. Oncol. 24 160-8

Liu H H, Koch N, Starkschall G, Jacobson M, Forster K, Liao Z, Komaki R and Stevens C W 2004 Evaluation of internal lung motion for respiratory-gated radiotherapy using MRI: part IImargin reduction of internal target volume Int. J. Radiat. Oncol. Biol. Phys. 60 1473-83

Lowe D G 2004 Distinctive image features from scale-invariant keypoints Int. J. Comput. Vis. 60 91-110

Mukumoto N et al 2014 Intrafractional tracking accuracy in infrared marker-based hybrid dynamic tumour-tracking irradiation with a gimballed linac Radiother. Oncol. 111 301-5

Murphy M J and Dieterich S 2006 Comparative performance of linear and nonlinear neural networks to predict irregular breathing Phys. Med. Biol. 51 5903-14

Mutic S and Dempsey J F 2014 The viewray system: magnetic resonance-guided and controlled radiotherapy Semin. Radiat. Oncol. 24 196-99

Paganelli C, Peroni M, Baroni G and Riboldi M 2013 Quantification of organ motion based on an adaptive image-based scale invariant feature method Med. Phys. 40111701

Paganelli C, Seregni M, Fattori G, Summers P, Bellomi M, Baroni G and Riboldi M 2015a Magnetic resonance imaging-guided versus surrogate-based motion tracking in liver radiation therapy: a

prospective comparative study Int. J. Radiat. Oncol. 91 840-48

Paganelli C, Lee D, Greer P B, Baroni G, Riboldi M and Keall P 2015b Quantification of lung tumor rotation with automated landmark extraction using orthogonal cine MRI images Phys. Med. Biol. $607165-78$

Plathow C, Ley S, Fink C, Puderbach M, Hosch W, Schmähl A, Debus J and Kauczor H U 2004 Analysis of intrathoracic tumour mobility during whole breathing cycle by dynamic MRI. Int. $J$. Oncol. Biol. Phys. 59 952-9

Radiat.

Plathow C, Klopp M, Fink C, Sandner A, Hof H, Puderbach M, Herth F, Schmähl A and Kauczor H U 2005 Quantitative analysis of lung and tumour mobility: comparison of two time-resolved MRI sequences Br. J. Radiol. 78 836-40

Plathow C, Schoebinger M, Herth F, Tuengerthal S, Meinzer H P and Kauczor H U 2009 Estimation of pulmonary motion in healthy subjects and patients with intrathoracic tumours using 3D-dynamic MRI: initial results Korean J. Radiol. 10 559-67

Ruan D 2010 Kernel density estimation-based real-time prediction for respiratory motion Phys. Med. Biol. 55 1311-26

Sawant A, Keall P, Pauly K B, Alley M, Vasanawala S, Loo B W Jr, Hinkle J and Joshi S 2014 Investigating the feasibility of rapid MRI for image-guided motion management in lung

cancer radiotherapy BioMed. Res. Int. 2014485067

Sharp G C, Jiang S B, Shimizu S and Shirato H 2004 Prediction of respiratory tumour motion for real-time image-guided radiotherapy Phys. Med. Biol. 49 425-40

Shirato H et al 2000 Physical aspects of a real-time tumour tracking system for gated radiotherapy Int. J. Radiat. Oncol. Biol. Phys. 48 1187-95

Tryggestad E, Flammang A, Hales R, Herman J, Lee J, McNutt T, Roland T, Shea S M, Wong J 2013 4D tumour centroid tracking using orthogonal 2D dynamic MRI: implications for

radiotherapy planning Med. Phys. 40091712

Verellen D, Depuydt T, Gevaert T, Linthout N, Tournel K, Duchateau M, Reynders T, Storme G and

De Ridder M 2010 Gating and tracking, 4D in thoracic tumours Cancer Radiother. 14 446-54

Wu H, Zhao Q, Berbeco R I, Nishioka S, Shirato H and Jiang S B 2008 Gating based on internal/external signals with dynamic correlation updates Phys. Med. Biol. 53 7137-50 